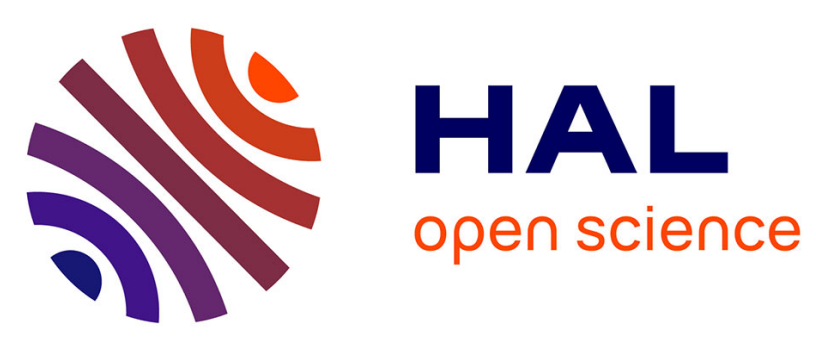

\title{
Nonlinear Adaptive Output Feedback Control of Series Resonant DC-DC Converters
}

Ouadia Elmaguiri, Fouad Giri, Luc Dugard, H. El Fadil, Fatima Zara Chaoui

\section{To cite this version:}

Ouadia Elmaguiri, Fouad Giri, Luc Dugard, H. El Fadil, Fatima Zara Chaoui. Nonlinear Adaptive Output Feedback Control of Series Resonant DC-DC Converters. ACC 2010 - American Control Conference, Jun 2010, Baltimore, Maryland, United States. pp.n.c. hal-00550226

\section{HAL Id: hal-00550226 https://hal.science/hal-00550226}

Submitted on 24 Dec 2010

HAL is a multi-disciplinary open access archive for the deposit and dissemination of scientific research documents, whether they are published or not. The documents may come from teaching and research institutions in France or abroad, or from public or private research centers.
L'archive ouverte pluridisciplinaire HAL, est destinée au dépôt et à la diffusion de documents scientifiques de niveau recherche, publiés ou non, émanant des établissements d'enseignement et de recherche français ou étrangers, des laboratoires publics ou privés. 


\title{
Nonlinear adaptive output feedback control of series resonant dc-dc converters
}

\author{
O. Elmaguiri, F.Giri*, L. Dugard ${ }^{* *}$, H. El Fadil, F.Z. Chaoui \\ GREYC lab, University of Caen, France \\ * Corresponding author: fouad.giri@unicaen.fr \\ ** GIPSA, ENSIEG, INPG, Grenoble, France
}

\begin{abstract}
The problem of regulating the output voltage of DC-to-DC series resonant converters (SRC) is addressed. The difficulty is threefold: (i) the converter model involves discontinuous and highly nonlinear terms and is, further, controlled through a modulating frequency signal; (ii) all state variables are not accessible to measurements; (iii) the load is uncertain and may even be varying. An output feedback controller, not necessitating the measurement of the converter state variables, is proposed and shown to ensure semi-global stabilization of the closed-loop system and perfect output reference asymptotic tracking. The controller is developed using the backstepping control approach and the high-gain observer design technique.
\end{abstract}

\section{INTRODUCTION}

Series and parallel resonant DC-to-DC converters, and their various variants, have been given a great deal of interest in the power electronic literature. Compared to (hard) switched converters, SRC converters present several advantages e.g. they provide much higher power supplies. As they do not involve switched components, power losses are considerably reduced improving thus the conversion efficiency. However, SRC converters are more complex to control as they involve much more nonlinear dynamics. Furthermore, they are supplied by bipolar square signal generators and, consequently, the switching frequency is in general the only available control variable. These considerations make SRC modeling a particularly hard task. A modeling approach, based on generalized averaging, was developed in [1]. Small signal models for series and parallel resonant converters were developed in [2].

In the present work, following the first harmonic approach [1], a fifth order state-space model is developed for the converter of fig (1). From the control design viewpoint, the difficulty lies in: (i) the system nonlinear and discontinuous nature; (ii) the fact that the control signal (switching frequency) comes in all state variable equations. (iii) the vector state is not completely measurable and it should be estimated. Different control strategies were proposed for the considered class of converters. These include hybrid flatness based control [3], resonant tanks variables based optimal control [4], sliding mode control [5] and passivity based control [6]. In the present work, a new control strategy is developed to cope with the problem of output voltage regulation in SRC converters without assuming the state variables to be measurable and the load to be known. Following [7], a high gain observer is first designed to get estimates of the state variables that are not accessible to measurements. Then, an adaptive output control law is designed, using the tuning functions backstepping technique [8], based on the above state observer. It is worth recalling that, unlike linear systems, the separation principle does not systematically apply to nonlinear systems [9]. Furthermore, a parameter projection will be introduced in the parameter adaptive law (estimating online the load) to prevent possible parameter estimate drift that, otherwise, could result due to the presence of state estimation errors. The output adaptive controller thus obtained is formally shown to achieve quite interesting performances. Specifically, the closed-loop system is asymptotically stable and the attraction region size can be made arbitrarily large by conveniently choosing the control design parameters. The output reference tracking error vanishes asymptotically. The unknown load is perfectly identified.

The paper is organized as follows: the studied series resonant converter is described and modeled in Section II; the state observer is presented in Section III; the adaptive output feedback controller is designed in Section IV and the resulting closed-loop system is analyzed in section $\mathrm{V}$; the controller performances are illustrated by simulation in Section VI; technical proofs are placed in the appendix.

\section{SERIES RESONANT CONVERTER MODELLING}

The studied series resonant DC-to-DC converter is illustrated by Fig 1. A state-space representation of the system is the following:

$$
\begin{aligned}
& L \frac{d i}{d t}=-v-v_{o} \operatorname{sgn}(i)+E \operatorname{sgn}(\sin (\omega t)) \\
& C \frac{d v}{d t}=i \\
& C_{0} \frac{d v_{o}}{d t}=a b s(i)-\frac{v_{o}}{R}
\end{aligned}
$$

where $v$ and $i$ denote the resonant tank voltage and current respectively; $v_{o}$ is the output voltage supplying the load (here a resistor $R$ ); the power source supplying the converter is characterized by a constant amplitude $E$ and a varying switching frequency $\omega$ (in $r d / s$ ); $L$ and $C$ designate respectively the inductance and capacitance of the resonant tank;

As the supply source amplitude $E$ is constant, the pulsation $\omega$ turns out to be the only possible control variable. 


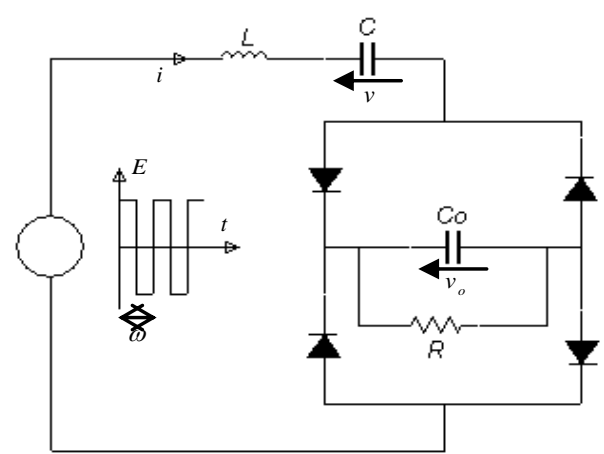

Fig 1. Series resonant converter under study

A control oriented model can be obtained applying to (1)-(3) the first harmonic approximation procedure introduced in [1]. Based upon the following assumption,

A1. The voltage $v$ and current $i$ are approximated with good accuracy by their (time varying) first harmonics (denoted $V_{1}$ and $I_{1} e^{j \Psi}$ respectively).

Doing so, one gets the following more convenient model: (see [1], [7] for details):

$\frac{d I_{1}}{d t}=-j \omega I_{1}+\frac{1}{L}\left[-V_{1}-\frac{2}{\pi} V_{0} e^{j \Psi}-j \frac{2 E}{\pi}\right]$

$\frac{d V_{1}}{d t}=-j \omega V_{1}+\frac{1}{C} I_{1}$

$\frac{d V_{o}}{d t}=\frac{4}{\pi C_{0}} a b s\left(I_{1}\right)-\frac{V_{o}}{R C_{o}}$

In the 'harmonic' model (4)-(6) the control signal $\omega$ comes in linearly. However, it still not suitable for control/observer designs because it involves complex variables and parameters. To get a convenient state-space model, introduce the following notations:

$$
I_{1}=x_{1}+j x_{2}, V_{1}=x_{3}+j x_{4}, V_{o}=x_{5}
$$

Substituting (7) in (4)-(6) yields the following state-space representation:

$$
\begin{aligned}
& \dot{x}_{1}=x_{2} u-\frac{x_{3}}{L}-\frac{2 x_{5}}{\pi L} \frac{x_{1}}{\sqrt{x_{1}^{2}+x_{2}^{2}}} \\
& \dot{x}_{2}=-x_{1} u-\frac{x_{4}}{L}-\frac{2 x_{5}}{\pi L} \frac{x_{2}}{\sqrt{x_{1}^{2}+x_{2}^{2}}}-\frac{2 E}{\pi L} \\
& \dot{x}_{3}=x_{4} u+\frac{x_{1}}{C} \\
& \dot{x}_{4}=-x_{3} u+\frac{x_{2}}{C} \\
& \dot{x}_{5}=\frac{4}{\pi C_{o}} \sqrt{x_{1}^{2}+x_{2}^{2}}-\frac{x_{5}}{C_{o}} \theta
\end{aligned}
$$

where $u=\omega$ and $\theta=1 / R$. The only quantities that are accessible to measurements are:

$$
x_{5}=V_{o}, \sqrt{x_{1}^{2}+x_{2}^{2}}=I_{1}, \sqrt{x_{3}^{2}+x_{4}^{2}}=V_{1}
$$

In [7] a high-gain observer has been designed to get accurate estimates of unmeasured variables and shown to be exponentially convergent if all system signals are bounded. This is defined using the following variable change:

$$
\begin{aligned}
& \Psi: I R^{5} \rightarrow I R^{8} ; \quad x \rightarrow z \\
& z_{1}=\sqrt{x_{1}^{2}+x_{2}^{2}} ; \quad z_{2}=\sqrt{x_{3}^{2}+x_{4}^{2}} ; z_{3}=x_{1} x_{3}+x_{2} x_{4} \\
& z_{4}=x_{2} ; z_{5}=x_{4} ; \quad z_{6}=x_{1} ; z_{7}=x_{3} ; z_{8}=x_{5}
\end{aligned}
$$

The equation describing the evolution of the new state variables, $z_{i}(i=1, \cdots, 7)$ is omitted for space limitation; it can be found in [7] where the following high gain observer was proposed:

$$
\begin{aligned}
& \dot{\hat{z}}_{1}=-\frac{\hat{z}_{3}^{2}}{L \hat{z}_{1}}-\frac{2 x_{5}}{\pi L}-\frac{2 E \hat{z}_{4}}{\pi L \hat{z}_{1}}-4 \lambda\left(\hat{z}_{1}-z_{1}\right) \\
& \dot{z}_{2}=\frac{\hat{z}_{3}}{C \hat{z}_{2}}-4 \lambda\left(\hat{z}_{2}-z_{2}\right) \\
& \hat{z}_{3}=-\frac{\hat{z}_{2}^{2}}{L}+\frac{\hat{z}_{1}^{2}}{C}-\frac{2 \hat{z}_{3} x_{5}}{\pi L \hat{z}_{1}}-\frac{2 E z_{5}}{\pi L}-6 \lambda^{2} C \hat{z}_{2}\left(\hat{z}_{2}-z_{2}\right) \\
& \hat{z}_{4}=-\hat{z}_{6} u-\frac{\hat{z}_{5}}{L}-\frac{2 x_{5} \hat{z}_{4}}{\pi L \hat{z}_{1}}-\frac{2 E}{\pi L}-6 \lambda^{2}\left(\frac{L \pi \hat{z}_{1}}{2 E}\left(\hat{z}_{1}-z_{1}\right)\right. \\
& \hat{z}_{5}=-\frac{C \pi \hat{z}_{2} u}{2 E}\left(\hat{z}_{2}-\frac{\left.z_{2}\right)}{C}+4 \lambda^{3} \frac{L C \pi \hat{z}_{2}}{2 E}\left(\hat{z}_{2}-z_{2}\right)\right. \\
& \hat{z}_{6}=\hat{z}_{4} u-\frac{\hat{z}_{7}}{L}-\frac{2 x_{5} \hat{z}_{6}}{\pi L \hat{z}_{1}}-\frac{2 E}{\pi L}-6 \lambda^{3}\left(\frac{L \pi \hat{z}_{1}}{2 E u}\left(\hat{z}_{1}-z_{1}\right)\right. \\
& \hat{\alpha}=\frac{C \hat{z}_{7}}{L^{4} \pi \hat{z}_{1}^{2}}+\frac{1}{\left(\pi L C \hat{z}_{2}\right)^{2}}\left(\hat{z}_{5} u+\frac{\hat{z}_{6}}{C}-\lambda^{4}\left(\frac{\left.\left(\hat{z}_{2}-z_{2}\right)\right)}{2 E \pi L C \hat{z}_{2} \hat{\alpha} u}-\frac{3\left(\hat{z}_{1}-z_{1}\right)}{2 E L^{2} \pi C \hat{z}_{1} \hat{\alpha} u}\right)\right. \\
& 9
\end{aligned}
$$

where $\lambda$ denotes a design parameter.

The convergence of the above observer has been analyzed in [7] using the following Lyapunov function:

$V_{o b}(\tilde{z})=\tilde{z}^{T} \Delta_{\lambda}^{T} S_{1} \Delta_{\lambda} \tilde{z}$

with

$\tilde{z}=z-\hat{z}$

$\Delta_{\lambda}^{-1}=\operatorname{diag}\left[\begin{array}{llll}I_{2 \times 2} & \lambda I_{2 \times 2} & \lambda^{2} I_{2 \times 2} & \lambda^{3} I_{2 \times 2}\end{array}\right]$

where $I_{2 \times 2}$ denotes the $2 \times 2$ identity matrix and $S_{1}$ is a symmetric positive definite matrix that is the unique solution of the Lyapunov equation:

$S_{1}+A^{T} S_{1}+S_{1} A-C^{T} C=0$

with $A$ and $C$ defined as follows: 


$$
A=\left[\begin{array}{cccc}
0 & I_{2} & 0 & 0 \\
0 & 0 & I_{2} & 0 \\
0 & 0 & 0 & I_{2} \\
0 & 0 & 0 & 0
\end{array}\right], \quad C=\left[\begin{array}{llll}
I_{2 \times 2} & O_{2 \times 2} & O_{2 \times 2} & O_{2 \times 2}
\end{array}\right]
$$

Theorem 1 ([7]). Consider the system (8)-(12), subject to Assumptions A1-A2, the state variable change (14a-c) and the state observer (15a-h). Suppose all the system and observer state variables to be bounded so that all involved nonlinearities can be supposed to be Lipschitz. Then, the time-derivative of $V_{o b}(\tilde{z})$ along the trajectory of $\tilde{z}$ satisfies the inequality

$\dot{V}_{o b} \leq-(\lambda-l) V_{o b}$

for some real constant $l>0$, depending on the Lipschitz coefficients of the different nonlinearities. Consequently, the state estimation error $\tilde{z}=z-\hat{z}$ converges exponentially to zero, whatever the initial condition $\hat{z}(0)$, provided the observer gain $\lambda$ is sufficiently large

\section{ADAPTIVE CONTROL DESIGN}

The load resistance $\mathrm{R}$ in model (1-3) is allowed to undergo infrequent jumps. To cope with such a parameter uncertainty the adaptive controller to be designed should involve an on line estimation of the unknown parameter $\theta=1 / R$. The unknown parameter estimate and the corresponding estimation error are denoted $\hat{\theta}$ and $\tilde{\theta}=\theta-\hat{\theta}$, respectively. Following closely (Kristic et al., 1995) the adaptive controller is designed in three major steps.

Design Step 1. Introduce the tracking error:

$$
e_{1}=x_{5}-x_{5 \text { ref }}
$$

where $x_{5 \text { ref }}$ denotes the desired constant output reference. Achieving the tracking objective amounts to enforcing the error $e_{1}$ to vanish. To this end, the $e_{1}$-dynamics need to be clearly defined. Deriving (20) one obtains:

$$
\dot{e}_{1}=\frac{4}{\pi C_{o}} z_{1}-\frac{x_{5}}{C_{o}} \theta
$$

The quantity $\frac{4}{\pi C_{o}} z_{1}$ stands as a virtual control input in (21). Consider the following Lyapunov equation:

$$
V_{c 1}\left(e_{1}, \tilde{\theta}\right)=\frac{1}{2} e_{1}^{2}+\frac{1}{2 \gamma} \tilde{\theta}^{2}
$$

where $\gamma>0$ is a design parameter, called adaptation gain.

Time-derivation of $V_{c 1}$, along the $\left(e_{1}, \tilde{\theta}\right)$-trajectory, is:

$$
\dot{V}_{c 1}=e_{1}\left(\frac{4}{\pi C_{o}} z_{1}+w_{1} \hat{\theta}\right)-\frac{\tilde{\theta}}{\gamma}\left(\dot{\hat{\theta}}-\gamma w_{1} e_{1}\right)
$$

where $w_{1}$ denotes the first regressor function defined by:

$$
w_{1}=-\frac{x_{5}}{C_{o}}
$$

one can eliminate $\tilde{\theta}$ from $\dot{V}_{1}$ using the law $\dot{\hat{\theta}}=\gamma \tau_{1}$ with:

$$
\tau_{1}=w_{1} e_{1}
$$

Furthermore, $e_{1}$ can be regulated to zero if $\frac{4}{\pi C_{o}} z_{1}=\alpha_{1}$

where the stabilizing function $\alpha_{1}$ is defined by:

$$
\alpha_{1}=-c_{1} e_{1}-w_{1} \hat{\theta}
$$

where $c_{1}>0$ is a design parameter. Since $4 z_{1} / \pi C_{o}$ is not the actual control input, we can only seek the convergence of the error $\left(4 z_{1} / \pi C_{o}\right)-\alpha_{1}$ to zero. Also, we do not take $\dot{\hat{\theta}}=\gamma \tau_{1}$ as parameter update law. Nevertheless, we retain $\tau_{1}$ as the first tuning function and tolerate the presence of $\tilde{\theta}$ in $\dot{V}_{1}$. Introduce the second error variable:

$$
e_{2}=\frac{4}{\pi C_{o}} z_{1}-\alpha_{1}
$$

Then, equation (21) becomes using (26) and (27):

$$
\dot{e}_{1}=-c_{1} e_{1}+e_{2}+w_{1} \tilde{\theta}
$$

Also, (26) can be rewritten as follows:

$\dot{V}_{c 1}=-c_{1} e_{1}^{2}+e_{1} e_{2}+\tilde{\theta}\left(\tau_{1}-\frac{\dot{\hat{\theta}}}{\gamma}\right)$

Design Step 2. The objective now is to make the error variables $\left(e_{1}, e_{2}\right)$ vanish asymptotically. To this end, the dynamics of $e_{2}$ are first determined. Deriving (27) one obtains, using (14a-c), (24) (26) and (28):

$$
\begin{aligned}
\dot{e}_{2}= & -\frac{8 E z_{4}}{\pi^{2} L C_{o} z_{1}}-\frac{4 z_{3}}{\pi L C_{o} z_{1}}-\frac{8 x_{5}}{\pi^{2} L C_{o}}-\frac{\hat{\theta}}{C_{o}}\left(\frac{4}{\pi C_{o}} z_{1}-\frac{x_{5}}{C_{o}} \hat{\theta}\right) \\
& +c_{1}\left(-c_{1} e_{1}+e_{2}\right)+w_{1} \dot{\hat{\theta}}+\tilde{\theta}\left(c_{1} w_{1}+\frac{x_{5}}{C_{o}^{2}} \hat{\theta}\right)
\end{aligned}
$$

As the states $z_{i}(i=3,4)$ are not available they are replaced in (30) by their estimates provided by (15c-d). Doing so, one gets:

$$
\begin{aligned}
\dot{e}_{2}=- & \frac{8 E \hat{z}_{4}}{\pi^{2} L C_{o} z_{1}}-\frac{4 \hat{z}_{3}}{\pi L C_{o} z_{1}}-\frac{8 x_{5}}{\pi^{2} L C_{o}}-\frac{\hat{\theta}}{C_{o}}\left(\frac{4}{\pi C_{o}} z_{1}-\frac{x_{5}}{C_{o}} \hat{\theta}\right) \\
& +c_{1}\left(-c_{1} e_{1}+e_{2}\right)+w_{1} \dot{\hat{\theta}}+\tilde{\theta}\left(c_{1} w_{1}+\frac{x_{5}}{C_{o}^{2}} \hat{\theta}\right) \\
& -\frac{8 E}{\pi^{2} L C_{o}} \frac{\tilde{z}_{4}}{z_{1}}-\frac{4}{\pi L C_{o}} \frac{\tilde{z}_{3}}{z_{1}}
\end{aligned}
$$

where $\tilde{z}_{3}$ and $\tilde{z}_{4}$ are the estimation errors of $z_{3}$ and $z_{4}$. Introduce the new error:

$e_{3}=-\frac{8 E \hat{z}_{4}}{\pi^{2} L C_{o} z_{1}}-\alpha_{2}$

Then (31) is rewritten as follows:

$\dot{e}_{2}=e_{3}+\alpha_{2}+\psi_{2}+w_{2} \tilde{\theta}+w_{1} \dot{\hat{\theta}}+\chi_{1}\left(z_{1}, \tilde{z}_{3}, \tilde{z}_{4}\right)$

where the second regression function is defined by: 
$w_{2}=c_{1} w_{1}+\frac{x_{5}}{C_{o}^{2}} \hat{\theta}$

and

$$
\begin{gathered}
\psi_{2}=-\frac{4}{\pi L C_{o} z_{1}} \hat{z}_{3}-\frac{8 x_{5}}{\pi^{2} L C_{o}}+c_{1}\left(-c_{1} e_{1}+e_{2}\right) \\
-\frac{\hat{\theta}}{C_{o}}\left(\frac{4}{\pi C_{o}} z_{1}-\frac{x_{5}}{C_{o}} \hat{\theta}\right) \\
\chi_{1}\left(z_{1}, \tilde{z}_{3}, \tilde{z}_{4}\right)=-\frac{8 E}{\pi^{2} L C_{o}} \frac{\tilde{z}_{4}}{z_{1}}-\frac{4}{\pi L C_{o}} \frac{\tilde{z}_{3}}{z_{1}}
\end{gathered}
$$

Notice that the disturbing term $\chi_{1}\left(z_{1}, \tilde{z}_{3}, \tilde{z}_{4}\right)$ vanishes exponentially fast whenever $\tilde{z}_{3}, \tilde{z}_{4}$ do so. Consider the augmented Lyapunov function:

$V_{c 2}\left(e_{1}, e_{2}, \tilde{\theta}\right)=V_{c 1}\left(e_{1}, \tilde{\theta}\right)+\frac{1}{2} e_{2}^{2}$

Its derivative along the solution of (20) and (27) is:

$$
\begin{aligned}
\dot{V}_{c 2} & =-c_{1} e_{1}^{2}+e_{2}\left(e_{1}+e_{3}+\alpha_{2}+\psi_{2}+w_{1} \dot{\hat{\theta}}\right) \\
& +\tilde{\theta}\left(\tau_{1}+e_{2} w_{2}-\frac{\dot{\hat{\theta}}}{\gamma}\right)+e_{2} \chi_{1}
\end{aligned}
$$

$\tilde{\theta}$ can be cancelled in $\dot{V}_{2}$ using the update law $\dot{\hat{\theta}}=\gamma \tau_{2}$ :

$\tau_{2}=\tau_{1}+\omega_{2} e_{2}=\left[\begin{array}{ll}w_{1} & w_{2}\end{array}\right]\left[\begin{array}{l}e_{1} \\ e_{2}\end{array}\right]$

If $-8 E \hat{z}_{4} /\left(\pi^{2} L C_{o} z_{1}\right)$ were the actual control in (31) and the term $\chi_{1}$ were null, one would get $\dot{V}_{c 2}=-c_{1} e_{1}^{2}-c_{2} e_{2}^{2}$ by using the above parameter update law and letting:

$\alpha_{2}=-e_{1}-\psi_{2}-c_{2} e_{2}-w_{1} \gamma \tau_{2}$

As $-8 E \hat{z}_{4} /\left(\pi^{2} L C_{o} z_{1}\right)$ is just a virtual control, the above parameter update law is not sufficient. Nevertheless, we retain $\tau_{2}$ as a second tuning function. Then, (38) gives:

$$
\begin{aligned}
\dot{V}_{c 2}= & -c_{1} e_{1}^{2}-c_{2} e_{2}^{2}-e_{2} w_{1}\left(\gamma \tau_{2}-\dot{\hat{\theta}}\right)+\tilde{\theta}\left(\tau_{2}-\frac{\dot{\hat{\theta}}}{\gamma}\right)+e_{2} e_{3} \\
& +e_{2} \chi_{1}\left(z_{1}, \tilde{z}_{3}, \tilde{z}_{4}\right)
\end{aligned}
$$

Design Step 3. Deriving (32) gives:

$$
\dot{e}_{3}=-\frac{8 E}{\pi^{2} L C_{o}}(\overbrace{\hat{z}_{4}}^{\dot{z}})-\dot{\alpha}_{2}
$$

On the other hand, one obtains from (14b) and (15d):

$$
\frac{\hat{z}_{4}}{z_{1}}=-\frac{\hat{z}_{6}}{z_{1}} u+\delta_{1}\left(z_{1}, z_{2}, \hat{z}\right)+\tilde{z}_{3} \frac{\hat{z}_{4}}{L z_{1}^{3}}+\tilde{z}_{4} \frac{2 E \hat{z}_{4}^{2}}{z_{1}^{3}}
$$

with

$$
\begin{aligned}
\delta_{1}=-\left[\frac{\hat{z}_{5}}{L}\right. & \left.+2 \frac{x_{5} \hat{z}_{4}}{\pi L z_{1}}+\frac{2 E}{\pi L}-\frac{\hat{z}_{4}}{z_{1}}\left(-\frac{\hat{z}_{3}}{L z_{1}}-\frac{2 x_{5}}{\pi L}-\frac{2 E}{\pi L z_{1}} \hat{z}_{4}\right)\right] \\
& +6 \lambda^{2}\left(\frac{L \pi \hat{z}_{1}}{2 E}\left(\hat{z}_{1}-z_{1}\right)-\frac{C \pi \hat{z}_{2}}{2 E}\left(\hat{z}_{2}-z_{2}\right)\right)
\end{aligned}
$$

Furthermore, it is readily seen from (40) that:

$$
\begin{gathered}
\dot{\alpha}_{2}=-\gamma\left[\dot{w}_{1} \tau_{2}+w_{1}\left(\dot{w}_{1} e_{1}+w_{1} \dot{e}_{1}+\dot{w}_{2} e_{2}+w_{2} \dot{e}_{2}\right)\right] \\
-\dot{e}_{1}-\dot{\psi}_{2}-c_{2} \dot{e}_{2}
\end{gathered}
$$

Using (24), (34), (35) and (42), the derivatives on the right side of (45) can be given the following more suitable:

$\dot{e}_{1}=e_{10}+w_{1} \tilde{\theta}$

$\dot{e}_{2}=e_{20}+w_{1} \dot{\hat{\theta}}+w_{2} \tilde{\theta}+\chi_{1}($.)

$\dot{w}_{1}=-\frac{x_{50}}{C_{o}}-\frac{w_{1}}{C_{o}} \tilde{\theta}$

$\dot{\psi}_{2}=a_{0}\left(z_{10}+\tilde{z}_{10}\right)+a_{1} \dot{x}_{5}-a_{2} \frac{\dot{\hat{\theta}}}{C_{o}}+c_{1}\left(-c_{1} \dot{e}_{1}+\dot{e}_{2}\right)-\frac{4 \dot{\hat{z}}_{3}}{\pi L C_{o} z_{1}}$

To alleviate the text, the exact expressions of the newly introduced quantities (i.e. $e_{10}, e_{20}, x_{50}, z_{10}, \tilde{z}_{10}, a_{0}, a_{1}$ and $a_{2}$ ) are placed in the Appendix A. Substituting (43) and (45) in (42), one obtains:

$\dot{e}_{3}=\frac{8 E \hat{z}_{6}}{\pi^{2} L C_{o} z_{1}} u+\delta_{2}(z, \hat{z})+w_{3} \tilde{\theta}+g_{3} \dot{\hat{\theta}}+\chi_{2}\left(e_{i: 1.3}, z, \tilde{z}\right)$

where $w_{3}$ denotes the last regressor function defined by:

$$
\begin{aligned}
w_{3}= & \left(1-c_{1}^{2}+\gamma w_{1}^{2}\right)+\left(c_{1}+c_{2}+\gamma w_{1} w_{2}\right) w_{2} \\
& -\gamma\left(\tau_{2}+w_{1} e_{1}+w_{1} e_{2}\left(c_{1}-\frac{\hat{\theta}}{C_{o}}\right)-C_{o} a_{1}\right) w_{1}
\end{aligned}
$$

and

$$
\begin{aligned}
& \delta_{2}=-\frac{8 E}{\pi^{2} L C_{o}} \delta_{1}+\left(1-c_{1}^{2}+\gamma w_{1}^{2}\right) e_{10} \\
&+a_{0} z_{10}-\frac{4}{\pi L C_{o} z_{1}} \dot{\hat{z}}_{3}+\left(c_{1}+c_{2}+\gamma w_{1} w_{2}\right) e_{20} \\
&-\frac{\gamma}{C_{o}} x_{50}\left(\tau_{2}+w_{1} e_{1}+w_{1} e_{2}\left(c_{1}-\frac{\hat{\theta}}{C_{o}}\right)-a_{1} C_{o}\right) \\
& g_{3}= w_{1}\left(c_{1}+c_{2}+\gamma w_{1} w_{2}\right)-\left(\frac{a_{2}}{C_{o}}+\gamma \frac{w_{1}^{2} e_{2}}{C_{o}}\right) \\
& \chi_{2}=-\frac{8 E}{\pi^{2} L C_{o}}\left(\tilde{z}_{3} \frac{\hat{z}_{4}}{L z_{1}^{3}}+\tilde{z}_{4} \frac{2 E \hat{z}_{4}^{2}}{\pi L z_{1}^{3}}\right)+\left(c_{1}+c_{2}+\gamma w_{1} w_{2}\right) \chi_{1} \\
&+a_{0} \tilde{z}_{10}
\end{aligned}
$$

Note that the actual control input $u$ appeared for the first time in (50). Notice also that the term in $\chi_{2}$ vanishes exponentially fast whenever the $\tilde{z}_{3}, \tilde{z}_{4}$ do so. Now, the goal is to find a control law $u$ and adaptive law for $\hat{\theta}$ so that the $\left(e_{1}, e_{2}, e_{3}, \tilde{\theta}\right)$ system is asymptotically stable. To this end, consider the augmented Lyapunov function candidate:

$V_{c 3}\left(e_{1}, e_{2}, e_{3}, \tilde{\theta}\right)=V_{c 2}\left(e_{1}, e_{2}, \tilde{\theta}\right)+\frac{e_{3}^{2}}{2}=\sum_{i=1}^{3} \frac{e_{1}^{2}}{2}+\frac{\tilde{\theta}^{2}}{2 \gamma}$

Using (41) and (50), the derivative of $V_{c 3}$ turns out to be: 


$$
\begin{aligned}
\dot{V}_{c 3}= & -c_{1} e_{1}^{2}-c_{2} e_{2}^{2}-e_{2} w_{1}\left(\gamma \tau_{2}-\dot{\hat{\theta}}\right)+e_{3}\left[\frac{8 E \hat{z}_{6}}{\pi^{2} L C z_{1}} u+\delta_{2}\right. \\
& \left.+g_{3} \dot{\hat{\theta}}+e_{2}\right]+\tilde{\theta}\left(\tau_{2}-\frac{\dot{\hat{\theta}}}{\gamma}+e_{3} w_{3}\right)+e_{3} \chi_{2}\left(e_{i: 1 . .3}, \tilde{z}, z\right)
\end{aligned}
$$

The term in $\tilde{\theta}$ can be canceled on the right side of (56) using the update law $\dot{\hat{\theta}}=\gamma \tau_{3}$ with

$\tau_{3}=\tau_{2}+e_{3} w_{3}=\left[\begin{array}{lll}e_{1} & e_{2} & e_{3}\end{array}\right]\left[\begin{array}{l}w_{1} \\ w_{2} \\ w_{3}\end{array}\right]=e \mathrm{~W}$

However, this update law (which is a gradient type) is not suitable because of its integral nature. The disturbing term $\chi_{2}(z, \tilde{z}, \hat{z})$ in (56) may cause the divergence the estimate $\hat{\theta}$. This issue is commonly coped with resorting to estimate projection on a convex bounded set including the true parameter. Let such convex be any interval $C=\left[-M_{0}, M_{0}\right]$ such that $M_{0} \geq|\theta|$. Practical choice of $M_{0}$ is not an issue as this may be arbitrarily large. The gradient algorithm with projection is then defined as follows (see e.g. [10]):

$\dot{\hat{\theta}}=P\left(\gamma \tau_{3}\right)$

where $\hat{\theta}(0)$ is chosen so that $\hat{\theta}^{2}(0) \leq M_{0}^{2}, P($.$) is the$ projection operator defined by:

$P\left(\gamma \tau_{3}\right)=\left\{\begin{array}{cc}\gamma \tau_{3} \text { if } \hat{\theta}^{2}<M_{0}^{2} \text { or if }\left(\hat{\theta}^{2}=M_{0}^{2} \text { and } \gamma \tau_{3} \hat{\theta} \leq 0\right) \\ 0 \quad \text { otherwise }\end{array}\right.$

It is readily seen that this adaptive law maintains the estimate $\hat{\theta}$ in the convex bounded $\operatorname{set} C$. More interestingly, the projection operator $P($.) is shown in many places to possess the following key property (e.g. [10]):

$-\tilde{\theta} P\left(\gamma \tau_{3}\right) \leq-\tilde{\theta} \gamma \tau_{3}$

The expression of $\dot{V}_{c 3}$ suggest the following control law:

$u=\frac{\pi^{2} L C_{o} z_{1}}{8 E \hat{z}_{6}}\left(-\delta_{2}-g_{3} \dot{\hat{\theta}}-e_{2}-c_{3} e_{3}+v\right)$

where $c_{3}>0$ is a new parameter and $v$ is an additional control action resorted to cope with the parameter adaptive law saturation. The following choice will prove to be useful:

$v=\left\{\begin{array}{lll}-\gamma w_{1} w_{3} e_{2} & \text { if } & \dot{\hat{\theta}}=\gamma \tau_{3} \\ 0 & \text { if } & \dot{\hat{\theta}}=0\end{array}\right.$

\section{CLOSED LOOP STABILITY ANALYSIS}

Substituting the right side of (60a) for $u(t)$ in (50) and putting the resulting equation together with (28),(33),(40) ,(42) and (58a), one gets the following equations describing the trajectories of the errors $\left(e_{i}, i=1,2,3\right)$ and $\tilde{\theta}$ :

$\dot{e}=\bar{A} e+w^{T} \tilde{\theta}+\bar{w}+\chi$
$\dot{\tilde{\theta}}=-\dot{\hat{\theta}}=-P\left(\gamma \tau_{3}\right)$

where $\bar{A}$ is a skew symmetric matrix defined by:

$\bar{A}=\left[\begin{array}{ccc}-c_{1} & 1 & 0 \\ -1 & -c_{2} & 1+\sigma_{23} \\ 0 & -1-\sigma_{23} & -c_{3}\end{array}\right],\left(\sigma_{23}=\gamma w_{1} w_{3}\right)$

and

$$
\begin{aligned}
& w=\left[\begin{array}{lll}
w_{1} & w_{2} & w_{3}
\end{array}\right] ; \quad e=\left[\begin{array}{lll}
e_{1} & e_{2} & e_{3}
\end{array}\right]^{T} \\
& \bar{w}=\left[\begin{array}{lll}
0 & -w_{1}\left(\gamma \tau_{2}-\dot{\hat{\theta}}\right) & v
\end{array}\right]^{T} ; \quad \chi=\left[\begin{array}{lll}
0 & \chi_{1}(.) & \chi_{2}(.)
\end{array}\right]^{T}
\end{aligned}
$$

In addition to equations (61)-(62), the closed-loop system is also described by the equation (omitted for space limitation) describing the evolution of the state estimation error $\tilde{z}=z-\hat{z}$. The performances of the system are analyzed in the next theorem using the Lyapunov function:

$V(e, \tilde{z}, \tilde{\theta})=V_{o b}(\tilde{z})+V_{c 3}(e, \tilde{\theta})$

Theorem 2([11]). (Main result). Consider the control system consisting of the SRC model (8)-(12) in closed-loop with the adaptive controller composed of the control law (60a-b), the parameter update law (59a-b) and the high gain observer defined by $(15 a-h)$. For any $\eta>0$, there exist $0<c_{\text {min }}, \lambda_{\text {min }}<\infty$ such that, if $\min \left(c_{1}, c_{2}, c_{3}\right)>c_{\text {min }}$, $\lambda>\lambda_{\min }$ and $V(e(0), \tilde{z}(0), \tilde{\theta}(0))<\eta$ then:

1) all closed-loop signals remain bounded and the state estimation error $\tilde{z}=z-\hat{z}$ vanishes exponentially fast,

2) the tracking error $e_{1}=x_{5}-x_{5 \text { ref }}$ vanishes asymptotically,

$3)$ the parameter estimate $\hat{\theta}$ converges to its true value $\theta$

The theorem shows that the propose output feedback controller ensure asymptotically stability of the closed-loop system. The stability is semi-global as the controller design parameters are dependent on the system initial conditions.

\section{SIMULATION RESULTS}

The performances of the proposed adaptive controller are illustrated through numerical simulations. The controlled system, have the numerical values of Table 1 . The DC voltage source is fixed to $E=20 \mathrm{~V}$. The adaptive output feedback controller is given the following design parameters that have proved to be convenient:

$$
\begin{aligned}
& \lambda=1 \times 10^{3}, c_{1}=14 \times 10^{2}, c_{2}=5 \times 10^{2}, c_{3}=8 \times 10^{2}, \\
& \gamma=1 \times 10^{-13} \text { and } M_{0}=10 .
\end{aligned}
$$

Table 1: numerical values of the SRC characteristics

\begin{tabular}{clcc}
\hline \hline parameter & Symbol & value & \\
& & & unit \\
\hline Inductor & $\mathrm{L}$ & $0.9 \times 10^{-3}$ & $\mathrm{H}$ \\
Capacitor & $\mathrm{C}$ & $130 \times 10^{-6}$ & $\mathrm{~F}$
\end{tabular}




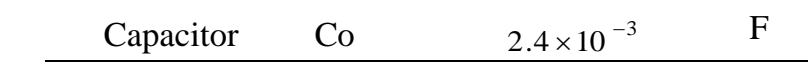

The initial states of $x$ and $\hat{z}$ are respectively:

$$
\begin{aligned}
x(0) & =\left[\begin{array}{llllllll}
0.35 & -0.75 & -5 & -8 & 5
\end{array}\right]^{T} \\
\hat{z}(0) & =\left[\begin{array}{llllllll}
0.3 & 0.3 & 0.2 & 0 & 0 & 0.25 & 0.5 & 0
\end{array}\right]^{T}
\end{aligned}
$$

The resulting control performances are illustrated by Figs. 2 to 4 . Fig 2a illustrates the closed-loop system responses to a step reference $x_{5 \text { ref }}(t)=8 v$ and successive converter load jumps. Specifically, the true load switches between $4.7 \Omega$ and $9 \Omega$ (Fig 2b). It is shown that the regulation objective is achieved after transient periods following load changes. Fig (2b) shows that the load estimate $\hat{\theta}^{-1}$ actually converges toward its varying true value $R$. Fig 3 shows that all state estimates converge to their true values after $5 \mathrm{~ms}$.

\section{CONCLUSION}

The problem of controlling series resonant converters has been addressed. An adaptive output feedback controller has been designed using the backstepping control technique and the high-gain observation approach. It is the first time that a controller, not necessitating the measurement of the state variables and the knowledge of the load, guarantees semiglobal stabilization and perfect output reference tracking for this class of converters.

\section{APPENDIX A. Expressions of auxiliary variables}

$$
\begin{array}{ll}
e_{10}=-c_{1} e_{1}+e_{2} ; \quad e_{20}=-e_{1}-c_{2} e_{2}+e_{3}-\gamma w_{1} \tau_{2} \\
x_{50}=\frac{4}{\pi C_{o}} z_{1}+w_{1} \hat{\theta} ; & z_{10}=\frac{-\hat{z}_{3}}{L z_{1}}-\frac{2 x_{5}}{\pi L}-\frac{2 E \hat{z}_{4}}{\pi L z_{1}} \\
\tilde{z}_{10}=\frac{-\tilde{z}_{3}}{L z_{1}}-\frac{2 E \tilde{z}_{4}}{\pi L z_{1}} ; & a_{0}=\frac{4}{\pi L C_{o}} \frac{\hat{z}_{3}}{z_{1}^{2}}-\frac{4 \hat{\theta}}{\pi C_{o}^{2}} \\
a_{1}=\frac{-8}{\pi^{2} L C_{o}}+\frac{\hat{\theta}^{2}}{C_{o}^{2}} ; & a_{2}=\frac{4}{\pi C_{o}} z_{1}-\frac{2 x_{5}}{C_{o}} \hat{\theta}
\end{array}
$$

\section{REFERENCES}

[1] Sun J. and H. Grotstohen, "Averaged modeling and analysis of resonant converter”, IEEE PESC, pp 707-713, 1993.

[2] Lin J.L. and J.S. Lew, "Analysis, modeling and robust controller design for a series resonant converter", IEEE IECON, vol 12, pp 665-670, 1996.

[3] Sira-Ramirez H. and R. Silva-Ortega, "On the control of the resonant converters: a hybrid flatness approach", $15^{\text {th }}$ International Symposium on Mathematical Theory of Networks and Systems, South Bend, August, 2002.

[4] Oruganti R., J.J. Young, F.C Lee. 'Implementation of optimal trajectory control of series resonant converters". IEEE Trans. Electron., vol 13, pp 318-327, 1998.

[5] Sosa A., J.L. Castilla, M. De Vicuna, L.G. Miret, J. Cruz. Sliding mode control for the fixed-frequency series resonant converter with asymmetrical clamped-mode modulation. IEEE ISIE, Vol 2, pp.675-680, 2005.

[6] Carasco J.M., G. Escobar, R. Ortega "Analysis and experimentation nonlinear dissipative controller for the series resonant converter" IEEE Trans. Power Electronics, vol. 15, No.3, pp.536-444, 2000.

[7] Giri F., F. Liu, O. El maguiri, H. El Fadil," Observation of state variables in resonant DC-DC converter using the high gain design approach" IFAC Symposium on Power Plants and Power Systems Control, 2009

[8] Krstic M., I. Kanellakopoulos and P.V. Kokotovic, "Nonlinear and adaptive control design", Willey, 1995.

[9] Atassi A. N. and H. K. Khalil, 'Separation results for the stabilization of nonlinear systems using different high-gain designs', Systems \& Control Letters., vol. 39, pp. 183-191, 2000.

[10] Ioannou P. and B. Fidan. 'Adaptive control tutorial'. SIAM, Philadelphia, 2006.

[11] O. Elmaguiri, F. Giri, H. El Fadil, F.Z. Chaoui. 'Nonlinear adaptive output feedback control of series resonant dc-dc converters'. Detailed version of the present work can be provided on request by the corresponding author.

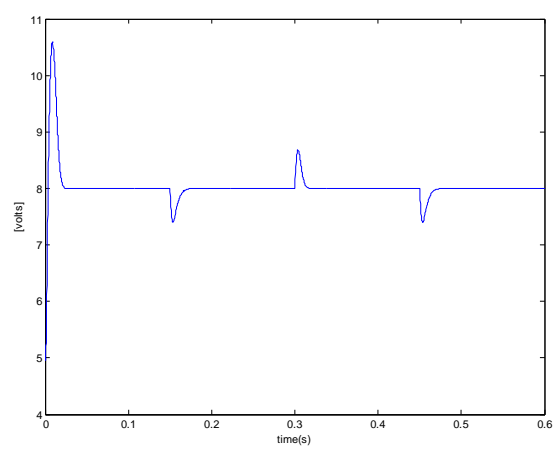

Fig 2a. Output voltage regulation in presence of varying converter load

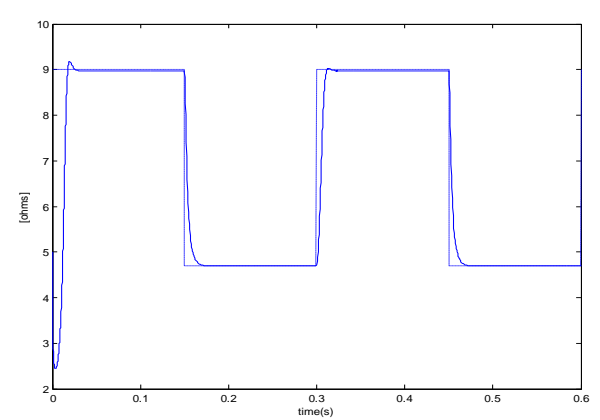

Fig 2b. Load estimate $\hat{\theta}^{-1}$ (solid) in presence of varying converter load $\theta^{-1}$ (dashed) 

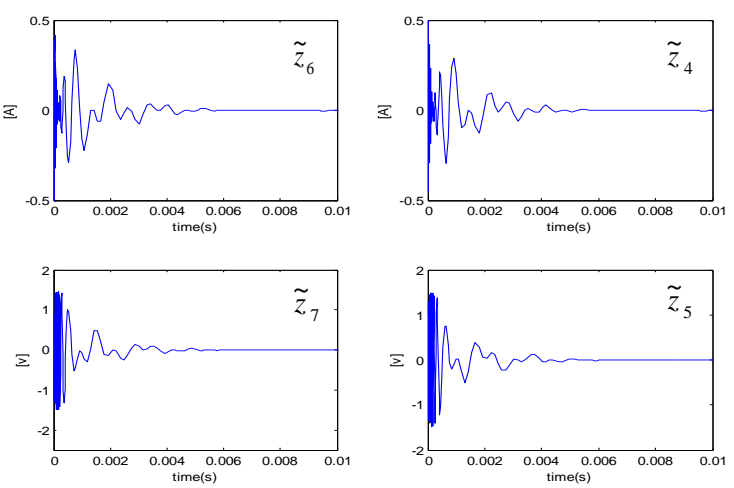

Fig 3: State estimation errors with $(\lambda=1000)$ 